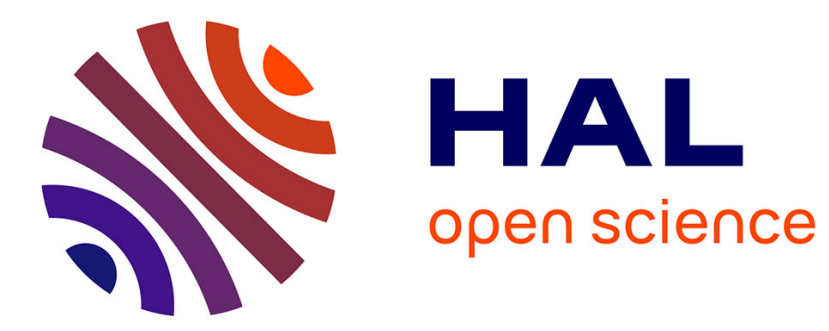

\title{
Effects of phonon dynamics on electrons in one-dimensional conductors
}

\author{
A. Bjelis, S. Barisic
}

\section{To cite this version:}

A. Bjelis, S. Barisic. Effects of phonon dynamics on electrons in one-dimensional conductors. Journal de Physique Lettres, 1975, 36 (6), pp.169-172. 10.1051/jphyslet:01975003606016900 . jpa-00231180

\section{HAL Id: jpa-00231180 https://hal.science/jpa-00231180}

Submitted on 1 Jan 1975

HAL is a multi-disciplinary open access archive for the deposit and dissemination of scientific research documents, whether they are published or not. The documents may come from teaching and research institutions in France or abroad, or from public or private research centers.
L'archive ouverte pluridisciplinaire HAL, est destinée au dépôt et à la diffusion de documents scientifiques de niveau recherche, publiés ou non, émanant des établissements d'enseignement et de recherche français ou étrangers, des laboratoires publics ou privés. 


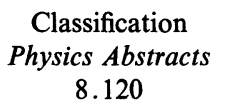

\title{
EFFECTS OF PHONON DYNAMICS ON ELECTRONS IN ONE-DIMENSIONAL CONDUCTORS
}

\author{
A. BJELIŠ and S. BARIŠIĆ \\ Institute of Physics, University of Zagreb \\ Croatia, Yugoslavia
}

(Reçu le 21 février 1975, accepté le 4 avril 1975)

\begin{abstract}
Résumé. - Nous étudions les électrons de conducteurs unidimensionnels, couplés aux phonons mous, décrits par un facteur de structure phénoménologique. Nous trouvons les critères exacts prouvant que le comportement critique de la densité d'états électronique dépend des indices critiques dynamiques du phonon mou. Nous discutons l'application de nos résultats aux matériaux réels.
\end{abstract}

\begin{abstract}
The exact conditions under which the critical behavior of the electron density of states involves the dynamical critical indices of the soft phonon, as well as the corresponding expressions for the electron density of states are derived for a model phonon structure factor. The application of these results to real materials is discussed.
\end{abstract}

A good understanding of the anomalous conductivity behavior in the quasi-one-dimensional systems requires a knowledge of simpler quantities, such as the density of states of the electron system. Beside this, the electron density of states is interesting in itself, since it is directly related to the magnetic susceptibility and other derived quantities [1-4]. These data show that the phonon softening produces progressively a dip in the density of states at the Fermi level. This dip merges into the Peierls gap at the lattice instability temperature.

An interesting problem which arises in this connection is the question of the phonon properties relevant to the electron density of states. Since the soft phonon modes become static at the transition temperature it is tempting to think [5-7] that only the static properties determine the forementioned dip in the density of states. While this is obviously true at the very transition temperature, we show that the behavior of the dip in the critical region can involve the dynamic phonon properties, i.e. the dynamical critical behavior. Among three limiting situations, which appear to be interesting in the model examined below, there is only one in which the static structure factor alone determines the electron spectrum. In this limit we recover the previously derived results [5-7].

At temperatures at which the thermal distribution of soft phonons becomes anomalous, the leading term in the first order (Migdal) approximation for electron self-energy is

$$
\begin{gathered}
\Sigma(p, \omega)=\frac{g^{2}}{2 \pi} \int_{-\infty}^{\infty} \mathrm{d} k \int_{-\infty}^{\infty} \mathrm{d} \omega^{\prime} S\left(2 p_{\mathrm{F}}-k, \omega^{\prime}\right) \times \\
\times G\left(p-2 p_{\mathrm{F}}+k, \omega+\omega^{\prime}\right) .
\end{gathered}
$$

Here $g^{2}$ is the electron-phonon coupling constant, $S\left(\omega^{\prime}\right)$ is the phonon dynamic structure factor and $G$ the (retarded) Green's function of one-dimensional electrons. In references $[6,7]$ this latter was replaced by its value at $\omega^{\prime}=0$. Then, after the use of KramersKronig relation, eq. (1) reduces to the starting point of reference [5], in which only the static phonon structure factor was involved from the outset. However this step is justified only if some general criteria on the functions $S(\omega)$ and $G$ are satisfied [8].

In order to illustrate this point we choose here the simple form of the dynamic structure factor

$$
\begin{aligned}
& S\left(2 p_{\mathrm{F}}-k, \omega\right)= \\
& \quad=2 \omega_{0} k_{\mathrm{B}} T \alpha \pi^{-1} /\left\{\left(l^{2} \omega^{2}-\omega^{2}\left(2 p_{\mathrm{F}}-k\right)\right)^{2}+\alpha^{2} \omega^{2}\right\},
\end{aligned}
$$

with the Lorentzian shape of the static structure factor

$$
\begin{aligned}
S\left(2 p_{\mathrm{F}}-k\right)= & \omega_{0} k_{\mathrm{B}} T / \pi \omega^{2}\left(2 p_{\mathrm{F}}-k\right)= \\
& =\omega_{0} k_{\mathrm{B}} T /\left(\pi \Omega^{2} .\left(1+\xi^{2} k^{2}\right)\right) .
\end{aligned}
$$


Here $\omega_{0}$ and $\Omega$ are respectively the bare and the renormalized phonon frequency at the Kohn wave number $2 p_{\mathrm{F}}$, and $\xi$ is the longitudinal temperature dependent correlation length. The dynamical parameters $\alpha$ and $l$ appearing in $S(\omega)$ are assumed to be real and independent of the wave vector. The parameter $l$ determines the shape of $S(\omega)$. For $l=0, S(\omega)$ reduces to the Lorentzian with the half-width

$$
\omega^{2}\left(2 p_{\mathrm{F}}-k\right) / \alpha \equiv \omega_{\mathrm{c}}\left(2 p_{\mathrm{F}}-k\right) .
$$

With $l$ arbitrary and for $\alpha>\sqrt{2} l \omega\left(2 p_{\mathrm{F}}-k\right), S(\omega)$ has a central peak structure, and is expected to describe situations in which the relaxation behavior is dominant [9]. In the limit $\alpha \gg l \omega\left(2 p_{\mathrm{F}}-k\right)$, the characteristic frequency of this central peak is $\omega_{\mathrm{c}}\left(2 p_{\mathrm{F}}-k\right)$, i.e. it becomes independent of $l$. In the opposite regime, $\alpha<\sqrt{2} l \omega\left(2 p_{\mathrm{F}}-k\right)$, the phonons are dispersive. The phonon group velocity in the Kohn anomaly is then equal to $\xi \Omega / l$. Obviously, this velocity should be smaller than the Fermi velocity $v_{\mathrm{F}}$, i.e. $l u \equiv v_{\mathrm{F}} l / \xi \Omega$ cannot be smaller than unity in the dispersive regime : $l u<1$ goes together with the overdamping $\alpha>\sqrt{2} l \Omega$.

After the integrations in complex $\omega^{\prime}$ and $k$ planes, eq. (1) reduces to

$$
\Sigma(x)= \begin{cases}\Delta /(x+i u \tilde{\Omega}) & l u>1 \\ \frac{\Delta}{x^{2}+u^{2} \widetilde{\Omega}^{2}}\left\{x+\frac{\tilde{\Omega}\left(\tilde{\alpha} u^{2}-i x\right)}{\left(l^{2}\left(x^{2}+u^{2} \widetilde{\Omega}^{2}\right)-\widetilde{\Omega}^{2}-u^{2} \tilde{\alpha}^{2}+2 \tilde{\alpha} x i\right)^{1 / 2}}\right\} & l u<1\end{cases}
$$

where the imaginary part of the square root in eq. (4b) has to be taken positive. The energy scale is conveniently defined by the parameter

$$
\Delta=\left(\omega_{0} k_{\mathrm{B}} T g^{2} / \Omega^{2} \xi\right)^{1 / 2},
$$

i.e. the reduced variables appearing in eq. (4) are $\widetilde{\Omega} \equiv \Omega / \Delta, \tilde{\alpha} \equiv \alpha / 2 \Delta$ and $x \equiv\left(\omega+v_{\mathrm{F}}\left(p-p_{\mathrm{F}}\right)\right) / \Delta$.

Depending on the value of $l u$, two different regimes are distinguished in eq. (5). For $l u>1, \Sigma(x)$ coincides with the static result $[5,6]$; whereas for $l u<1, \alpha$ and $l$ appear explicitly in the self-energy. Let us discuss the $x$-dependence of $\Sigma(x)$ in this dynamic regime. Remembering that $l u<1$ goes together with $\alpha \gtrsim l \Omega$, and assuming for simplicity $l u \ll 1$, we get

$$
\begin{aligned}
& \Sigma(x) \simeq \Delta(x-i \widetilde{\Omega} / l) / x^{2} \\
& \quad \text { for } \tilde{\alpha} / l^{2} \ll x, \\
& \Sigma(x) \simeq \Delta\left(x-i\left(\widetilde{\Omega}^{2}|x| / 4 \tilde{\alpha}\right)^{1 / 2}\right) / x^{2} \\
& \quad \text { for }\left(u^{2} \tilde{\alpha}^{2}+\widetilde{\Omega}^{2}\right) / \tilde{\alpha} \ll x \ll \tilde{\alpha} / l^{2},
\end{aligned}
$$

while for $x \ll\left(u^{2} \tilde{\alpha}^{2}+\tilde{\Omega}^{2}\right) / \tilde{\alpha}$ we distinguish two opposite limits :

$$
\begin{array}{lr}
\Sigma(x) \simeq \Delta /(x+i u \widetilde{\Omega}) & \text { if } \tilde{\alpha} u \gg \widetilde{\Omega}, \\
\Sigma(x) \simeq \Delta\left(-x l^{2}+3 x u^{2} \tilde{\alpha}^{2} /\left(x^{2}+u^{2} \tilde{\Omega}^{2}\right)-2 \tilde{\alpha i}\right) / 2 \widetilde{\Omega}^{2} \\
& \text { if } \tilde{\alpha} u \ll \widetilde{\Omega} .
\end{array}
$$

Note that in the case $(6 c) \Sigma(x)$ is of the static form $(4 a)$. The electron density of states is defined as

$$
\begin{aligned}
& N(\tilde{\omega}) / N_{0}= \\
& =-\pi^{-1} \int_{-\infty}^{\infty} \operatorname{Im}\left(2 \tilde{\omega}-x-\Delta^{-1} \Sigma(x)\right)^{-1} \mathrm{~d} x,
\end{aligned}
$$

where $\widetilde{\omega} \equiv \omega / \Delta$. Obviously, at the very instability $\left(\Omega=0, \omega_{\mathrm{c}}\left(2 p_{\mathrm{F}}\right)=0\right)$ the imaginary parts in eq. $(4 a, b)$ become infinitesimally small, while the real parts coincide and are equal to $\Delta / x$. The electron density of states has then the standard $\alpha, l$-independent form [5]

$$
N(\tilde{\omega}) / N_{0}= \begin{cases}0 & |\tilde{\omega}|<1 \\ |\tilde{\omega}| /\left(\tilde{\omega}^{2}-1\right)^{1 / 2} & |\tilde{\omega}|>1,\end{cases}
$$

whith $\Delta$ representing the half-width of the gap.

The critical behavior of parameters $\tilde{\alpha}$ and $u$ determines the manner in which $N(\widetilde{\omega}) / N_{0}$ tends to the form (8) when $\Omega \rightarrow 0$. There are three extreme possibilities related to different critical behaviors of $\tilde{\alpha}$ and $u$. They correspond to the cases when one of the regimes $(6 a-c)$ dominates in the range of integration in eq. (7). For brevity we quote only the results for $N(0)$ and $N(1)$; if $k_{\mathrm{B}} T<\Delta, N(0)$ is roughly equal to the thermally averaged density of states.

If $\tilde{\alpha} / l^{2} \ll 1$ (together with $l u<1$ ), $\Sigma(x)$ has the form given by eq. $(6 a)$, and we obtain

$$
N(0) / N_{0} \simeq \tilde{\Omega} / 2 l, \quad N(1) / N_{0}=1 / 2\left(N(0) / N_{0}\right)^{1 / 2} .
$$

If $\tilde{\alpha} / l^{2} \gg 1$ and $\tilde{\alpha} u^{2} \ll 1$, the decisive behavior is that given by eq. ( $6 b)$, and we find

$$
N(0) / N_{0} \simeq \widetilde{\Omega}_{\mathrm{c}}^{1 / 2} / 4, \quad N(1) / N_{0}=1 / 2^{5 / 4}\left(N(0) / N_{0}\right)^{1 / 2},
$$

where $\tilde{\Omega}_{\mathrm{c}} \equiv \omega_{\mathrm{c}}\left(2 p_{\mathrm{F}}\right) / \Delta$.

If $\tilde{\alpha} / l^{2} \gg 1$ and $\tilde{\alpha} u^{2} \gg 1$, the dominant contribution in eq. (7) comes from the static form $(6 c)$. This leads to

$$
N(0) / N_{0} \simeq \tilde{\Omega} u / 2, \quad N(1) / N_{0}=1 / 2\left(N(0) / N_{0}\right)^{1 / 2} .
$$

Eq. (9c) is just the $\Omega \rightarrow 0$ limit of the result first derived for soft phonons by Lee et al. [5]. For $l u>1$, this result holds for any $\tilde{\alpha}$. The limit $(6 d)$ does not 
correspond to the critical behavior for $\tilde{\alpha}$ and $u$ described above. This limit, as well as the intermediate cases $\tilde{\alpha} / l^{2} \simeq 1$ and $\tilde{\alpha} u^{2} \simeq 1$ in the critical limit, will be discussed in the more extended text [8].

In summary, if $\tilde{\alpha} u^{2} \sim \alpha / T^{1 / 2} \Omega \xi^{3 / 2} \gg 1$ the pseudogap in the electron spectrum is governed solely by static properties. In the opposite limit,

$$
\alpha / T^{1 / 2} \Omega \xi^{3 / 2} \ll 1,
$$

and if $l u<1$, the decrease with temperature of the density of states at the Fermi level is faster than that which is obtained from the static calculations. The corresponding critical behavior, defined by eq. $(9 a, b)$ is influenced, not only by the static and the dynamic critical exponents, but also by the shape $l$ of the dynamic structure factor.

We have also calculated [8] the electron selfenergy using the three-dimensional Lorentzian instead of eq. (3) in the whole range of integration in eq. (1). Provided that $\Omega \xi_{\perp} / k_{\mathrm{B}} T<a / \pi$, where $\xi_{\perp}$ is the transverse correlation length and a the interchain distance, the cut-off in the integration over transverse components of wave vector coincides with the Brillouin zone boundary [7]. Then, as long as

$$
\frac{\pi v_{\mathrm{F}}}{2 a \Delta} \frac{\xi_{\perp}}{\xi}\left(\left(1+\pi^{2} \xi_{\perp}^{2} / a^{2}\right)^{1 / 2}-1\right)^{1 / 2} \lesssim 1,
$$

$N(\tilde{\omega}) / N_{0}$ retains the one-dimensional form, but with the halfwidth of pseudo-gap given by

$$
\Delta_{\perp}=\Delta\left(\left(1+\pi^{2} \xi_{\perp}^{2} / a^{2}\right)^{1 / 2}-1\right)^{1 / 2} \sqrt{2} a / \pi \xi_{\perp} .
$$

Its critical behavior in the static regime (defined as before) is then given by

$$
N(0) / N_{0} \simeq(u \tilde{\Omega} / 2) \Delta / \Delta_{\perp}
$$

instead of eq. (9c). The dynamical results $(9 a, b)$ remain entirely unmodified (with $\Delta$ given by eq. (5)) if

$$
\xi_{\perp} / \xi \lesssim a u\left|\Delta_{\perp}^{2} l^{2}-u^{2} \alpha^{2}-2 \alpha \Delta_{\perp} i\right|^{1 / 2} / v_{\mathrm{F}} .
$$

is also satisfied.

Clearly, the requirement $\xi_{\perp} / \xi \ll 1$ is contained in the above condition (10). When this condition ceases to be satisfied, the electron density of states is dominated by $\xi_{\perp}$, i.e. the effects of phonon three-dimensionality become all important, as obtained previously for the static calculations [7]. A similar analysis applies to the case $\xi_{\perp} \Omega / k_{\mathrm{B}} T \gtrsim a / \pi$, i.e. to the transverse cut-off smaller than the Brillouin zone boundary. Again, the density of states remains essentially onedimensional provided that the ratio $\xi_{\perp} / \xi$ is small enough.

In the remaining text we wish to discuss how these results apply to real systems. The above discussion shows that the interchain coupling affects the electronic properties differently than the vibrational properties. This is not unexpected since only phonons are critical degrees of freedom. The one-dimensional approximation can be reasonably used on the electronic level provided that $\xi_{\perp} / \xi$ is small enough. However, even when $\xi_{\perp} / \xi \ll 1$ the phonon parameters in eq. $(2,3)$ will behave quite differently, according to the value of the ratio $\xi_{0 \perp} / a[10-13]$, where $\xi_{0 \perp}$ is the temperature independent transverse correlation length :

(i) $\xi_{0 \perp} \ll a$. The transverse cut-off in the calculation of phonon fluctuations is fixed by the Brillouin zone. Then, well above the critical temperature $T_{\mathrm{c}}$ the phonon parameters follow the one-dimensional fluctuation laws. In this regime the static fluctuation results [14] suggest that $\Omega^{2} \sim T / \xi$. Hence $u \sim(T \xi)^{-1 / 2}$ and $\tilde{\alpha} u^{2} \sim \alpha / T \xi$. This can be further specified by distinguishing, after reference [15], the cases of the scalar and complex order parameters.

In the first case both $\xi^{-1}$ and $u$ vanish exponentially with temperature [14]. For small $T_{\mathrm{c}}$, regardless of the value of $l$ this brings us into the regime $(4 b)$ for $\Sigma(x)$. The $T \rightarrow 0$ limit of $N(\tilde{\omega}) / N_{0}$ is of the standard form (9c) only if the divergence of $\alpha$ is stronger than that of $T \xi$.

In the second case, $\xi^{-1} \sim T$ [14], and $u$ depends only upon the non critical parameters (such as $v_{F}$ and those appearing in $\xi$ and $\Omega$ ). Depending on parameters, the $T \rightarrow 0$ value of $l u$ may be larger or smaller than one. If $l u<1$, and for small $T_{\mathrm{c}}$, the dynamical limit $(9 a, b)$ occurs if $\alpha \rightarrow 0$ when $T \rightarrow 0$; otherwise one recovers the standard result $(9 c)$.

Unfortunately, the theoretical knowledge of the temperature dependence of $\alpha$ is rather poor in onedimensional regime, and its experimental determination is desirable for further discussion.

Close above $T_{\mathrm{c}}$ the system crosses-over to the three-dimensional behavior with $\xi \simeq \xi_{\perp}[16]$.

(ii) $\xi_{0 \perp} \gg a$. The transverse cut-off in phonon calculations is fixed by $\xi_{0 \perp}^{-1}$. The parameters in eq. $(2,3)$ exhibit essentially three-dimensional critical laws, but $\xi_{\perp} / \xi=\xi_{0 \perp} / \xi_{0}(\lesssim 1)[10]$. In this case the one-dimensional results for $N(\widetilde{\omega}) / N_{0}$ (with the above mentioned modifications) can be valid in the whole temperature range above $T_{\mathrm{c}}$. The behaviors above and below $T_{\mathrm{c}}$, being of the same dimensionality can be related by the scaling laws. The dynamic scaling for $\alpha$ can be attempted. Using the analogy between the phase fluctuations [17] below the Peierls transition and e.g. the second sound in the superfluid helium [18], we expect that $\alpha$ vanishes when the system approaches the instability. This leads to the dynamic behavior of $N(\tilde{\omega}) / N_{0}$, analogous to that given by eq. $(9 a, b)$.

Well above $T_{\mathrm{c}}$ the anisotropic three-dimensional Kohn anomaly can be described within the meanfield approximation [19]. In this case

$$
\begin{gathered}
u \simeq\left(k_{\mathrm{B}} T / g\right)\left(8 \pi v_{\mathrm{F}} / \omega_{0}\right)^{1 / 2}, \quad l^{2} u^{2}=u^{2}-1, \\
\alpha / k_{\mathrm{B}} T \simeq 2 \pi / u^{2}
\end{gathered}
$$


and

$$
\tilde{\alpha} u^{2} \simeq\left(4 \pi^{2}\left(T-T_{\mathrm{c}}\right) / T_{\mathrm{c}}\right)^{1 / 4}
$$

Due to the vanishing of this last parameter, $N(\omega) / N_{0}$ is given by the (modified) results $(9 a, b)$, provided that $l u<1$. The departure of $l^{2}$ from unity is caused by the frequency dependence of the RPA phonon self-energy. This dependence is unimportant for $u \gtrsim 1$ [19-21]. For $u \lesssim 1$ the dynamic effects in the phonon renormalization become all important and lead to the three-peak form of the structure factor $S(\omega)[19,20]$. The $\omega$-dependence of the central peak is then given by eq. (2) with negative values of the parameter $l^{2}$. We shall discuss this range in the more extended text [8].

In conclusion, we have examined the electron density of states, using the simple phenomenological expression for the dynamic phonon structure factor.
We have derived the conditions under which the dynamic critical behavior of phonons governs the critical behavior of the density of states. It was further argued that the transversal components in the phonon structure factor can be neglected in the electronic calculation even when this is not possible in the calculations of the phonon critical properties. This observation leads to the large variety of the possible critical behaviors of the coefficients in the phonon structure factor involved in the electronic calculations. The temperature behaviors of the less known dynamic coefficients which lead to the dynamics-dominated electron density of states are determined using the usual critical laws for the static coefficients. When dynamic scaling may be used to describe the critical behavior of the soft phonon, the dynamics-dominated electron density of states is predicted.

We wish to acknowledge useful discussions with Prof. J. Friedel and Dr. H. Launois.

\section{References}

[1] Niedoba, H., Launois, H., Brinkmann, D. and Keller, H. U., J. Physique Lett. 35 (1974) L-251.

[2] Tomkiewicz, Y., Scott, B. A., TAO, L. J. and Titre, R. S., Phys. Rev. Lett. 32 (1974) 1363.

[3] Scott, J. C., Garito, A. F. and Hegger, A. J., Phys. Rev. B 10 (1974) 3131.

[4] Jacobsen, C. S., Tanner, D. B., Garito, A. F. and Heeger, A. J., Phys. Rev. Lett. 33 (1974) 1559.

[5] Lee, P. A., Rice, T. M. and Anderson, P. W., Phys. Rev. Lett. 31 (1973) 462.

[6] Patton, B. R. and Sham, L. J., Phys. Rev. Lett. 31 (1973) 631.

[7] Rice, M. J. and Strässler, S., Solid State Commun. 13 (1973) 1389.

[8] BJELIš, A. and BarIšlć, S., to be published.

[9] Renker, B., Pintschovius, L., Gläser, W., Rietschel, H., Comes, R., LieBert, L. and DreXel, W., Phys. Rev. Lett. 32 (1974) 836.

AXE, I. D. and ShIRANe, G., Phys. Rev. B 8 (1973) 1965.

[10] Barišić, S. and MarČElJa, S., Solid State Commun. 7 (1969) 1395.

[11] BarišIć, S. and Uzelac, K., J. Physique, 36 (1975) 325.
[12] Horovitz, B., GUtFreund, H. and Weger, M., to be published.

[13] BARIŠIĆ, S. and ŠAUB, K., to be published.

[14] Scalapino, D. J., Sears, M. and Ferrell, R. A., Phys. Rev. B 6 (1972) 3409.

[15] Toulouse, G., Nuovo Cimento 23B (1974) 234.

[16] Dieterich, W., Z. Phys. 270 (1974) 234.

[17] Lee, P. A., Rice, T. M. and Anderson, P. W., Solid State Commun. 14 (1974) 703.

[18] Ferrell, R. A., Menyhard, N., Schmidt, H., Schwabl, F. and Szepfalusy, P., Ann. Phys. 47 (1968) 565.

Brochard, F., J. Physique 34 (1973) 411.

[19] Barišıč, S., BJeliš, A. and ŠAuB, K., Solid State Commun. 13 (1973) 1119 ;

Bjeliš, A., ŠAUB, K. and Barišić, S., Nuovo Cimento 23B (1974) 102.

[20] Horovitz, B., Weger, M. and Gutfreund, H., Phys. Rev. B 9 (1974) 1246 ;

Horovitz, B., Gutrreund, H. and Weger, M., Solid State Commun. 11 (1972) 1361.

[21] Allender, D., Bray, J. W. and Bardeen, J., Phys. Rev. B 9 (1974) 119. 